American Journal of Infectious Diseases 4 (3): 193-199, 2008

ISSN 1553-6203

(C) 2008 Science Publications

\title{
Histological Features and ki-67 Index In Cervical Atypical Lesions
}

\author{
${ }^{1}$ Cabibi, D., ${ }^{2}$ L. Giovannelli, ${ }^{1}$ M. Cacciatore, ${ }^{1} \mathrm{C}$. Tripodo, ${ }^{2} \mathrm{P}$. Ammatuna, \\ ${ }^{1}$ F. Aragona and ${ }^{1}$ T. Gueli Alletti \\ ${ }^{1}$ Dipartimento di Patologia Umana, Università degli studi, Policlinico, Palermo \\ ${ }^{2}$ Dipartimento di Igiene e Microbiologia, Università degli studi, Policlinico, Palermo
}

\begin{abstract}
One of the most frequent challenges in routine diagnostic assessment of cervical biopsies consists of some lesions in which histological features of HPV infection are either inconsistently present or only mildly/focally evident. We named them Atypical Lesions (AL) because differential diagnosis with metaplastic/reactive lesions and LSIL is difficult. It would be important to identify among them those lesions that are really HPV-associated. We studied $52 \mathrm{AL}$ cases to evaluate whether the morphological features and the proliferation index, assessed by Mib1 (Ki67) immunostaining, would be useful to better understand the real nature of AL, with relation to the HPV presence and to the proliferative activity HPV induced. By univariate analysis, koilocytosis was found to be the only relatively sensitive and specific morphologic parameter associated with HPV status (74\% sensitivity, $72 \%$ specificity, $77.0 \%$ PPV). Mib1 index was not proven to be either a sensitive or a specific method (40.7\% sensitivity, $64.0 \%$, specificity and $55.0 \%$ PPV). Nevertheless, when AL were stratified by the three investigated features (koilocytosis, Mib1 immunostaining and HPV-DNA presence), they could be divided in subgroups consisting of normal cases (lacking of all the three studied features) reactive/repair changes, mimics of koilocytosis, latent, sub-clinical infection, cytopathic infection, without replicative activity (all of them at least lacking of one of the studied features) and finally, proliferative infection cases, in which all the three features were present. The last group showed high probability to harbour HR-HPV DNA (91\% of cases). In conclusion, AL appear as a very heterogeneous group of lesions, in which Mib1 assessment alone is not sensitive and specific enough to distinguish HPV negative from HPV positive lesions. Nevertheless, the Mib1 together with koylocitosis, could be useful to identify cases with HPV infection and increased proliferative activity that are at high risk of harbouring HR-HPV DNA and that could have a greater clinical rilevance.
\end{abstract}

Key words: HPV, koilocytosis, Mib1, PCR

\section{INTRODUCTION}

The most frequently detected alterations of squamous cervical epithelia consist of metaplastic/reactive conditions and human papillomavirus (HPV)-related dysplastic lesions, these latter being traditionally identified as cervical intraepithelial neoplasia CIN1, 2 or $3^{[1]}$. Recently, HPV associated lesions have been classified in the Bethesda System as Low-grade Squamous Intraepithelial Lesions (LSIL), which include CIN1 lesions and High-grade SIL (HSIL), which include CIN2 and CIN3. Whilst the intra-observer and inter-observer reproducibility reported in the assessment of HSIL is high, it is poor in LSIL $^{[2-9]}$. One likely reason is that also experienced histopathologists sometimes diagnose as LSIL some less striking HPV-induced lesions, which are not characterized by clear dysplasia and thus should not be considered as $\mathrm{CIN}^{[10]}$.

Some authors ${ }^{[8,11,12,13]}$ described in detail some of these lesions which, for the aim of this analysis, are hereafter referred to as Atypical Lesions (AL). In AL, histological features of HPV infection ${ }^{[11,14,15]}$ (namely, koilocytosis, multinucleation, acanthosis, papillomatosis, parakeratosis, individual cell keratinisation, mitosis in the lower basal third of the epithelium, hyperplasia of basal layers and absence of distinct basal cell layer) are either inconsistently present or only mildly/focally evident. Moreover, from the colposcopical point of view, both $\mathrm{AL}$ and metaplastic/reactive lesions and LSIL appear like acetowhite foci, which are usually bioptised and represent one of the most frequent challenges in routine surgical pathology practice.

Corresponding Author: Daniela Cabibi, Dipartimento di Patologia Umana, Università degli Studi, Palermo Via del Vespro, 129 Palermo Tel: +390916553533 Fax: +390916553549 
Similarly to the corresponding cytological category of atypical squamous cells of undetermined significance, which represents an equivocal cervical cytological diagnosis ${ }^{[16]}$, HPV testing could help to clarify the clinical significance of AL. Indeed, presence of high-risk (HR) oncogenic viral types (e.g., HPV-16, 18) would confer a different clinical importance, compared to Low-Risk (LR) types (e.g., HPV-6, -11). HPV testing, however, is presently too expensive and time consuming to be performed in the routine assessment of AL.

Recently, some adjutant methods have been considered in the histological assessment of epithelial lesions of the uterine cervix ${ }^{[17,18]}$. Among these, the cell proliferation-associated nuclear antigen Ki-67 has been taken into account. The Ki-67 protein is present in the G1, S, G2 and M phases of the cell cycle, while is not present in resting cells (G0 phase), therefore, Ki-67 testing by the monoclonal antibody Mib1 can provide an excellent index of the cell growth fraction. Since HPV-associated lesions show increased proliferative activity, the monoclonal antibody Mib1 might be a useful indicator of HPV presence.

The objective of the present study was to evaluate whether morphological features and Ki67 index (Mib1) would be able to stratify AL in sub-groups with different clinical meaning, with regard to presence of HPV and proliferative activity.

\section{MATERIALS AND METHODS}

Fifty-two consecutive formalin-fixed, paraffinembedded cervical biopsies morphologically consistent with AL were retrieved from archival material (January 2005-May 2005). All of them had been performed on colposcopically evidenced aceto-white foci and consisted of cases lacking of alterations required to qualify the lesion as $\mathrm{CIN} 1^{[11]}$, in which the features of HPV infections were either inconsistently present or only mildly/focally evident.

All cases showed histological alterations raising the question of differential diagnosis between HPVassociated LSIL and repair/reactive or metaplastic changes. On haematoxylin-eosin stained slides, the following morphological features were investigated, without knowledge of HPV status: koilocytosis, binucleation, acanthosis, papillomatosis, parakeratosis, mitosis in the lower third of epithelium, hyperplasia of parabasal layers, presence of distinct basal cell layer. They were coded as $0=$ absence, $1=$ presence.

For immunohistochemical assessment, tissue sections were treated using a microwave epitope retrieval technique with $10 \mathrm{mmol} \mathrm{L}^{-1}$ citrate buffer, $\mathrm{pH}$
6.0, at high temperature for $20 \mathrm{~min}$. Sections were then incubated with antibody against Ki-67 and coloured by the avidin-biotin-peroxidase complex method. Mib1 expression was assessed based on the presence of at least two stained epithelial nuclei in the same HPF (High Power Fields: each HPF identifies the area of the microscopic field evaluated by a $40 \mathrm{X}$ lens; $1 \mathrm{HPF}=$ $0.159 \mathrm{~mm} 2$ ) in the upper two thirds of cervical epithelium $^{[19,20]}$ and was coded as presence (+) /absence (-). The parabasal cells of squamous epithelium served as an internal positive control.

HPV detection and typing. Cervical biopsy specimens cut in $5 \mu \mathrm{m}$ sections were analysed. To isolate DNA, 2 sections were incubated overnight at $56^{\circ} \mathrm{C}$ in $250 \mu \mathrm{L}$ of a $0.5 \%$ Tween $20,50 \mathrm{mM}$ Tris- $\mathrm{HCl}$ $\mathrm{pH} 8.5,1 \mathrm{mM}$ EDTA, containing $300 \mu \mathrm{g} \mathrm{mL} \mathrm{m}^{-1}$ of proteinase $\mathrm{K}$ solution. Proteinase $\mathrm{K}$ was inactivated at $95^{\circ} \mathrm{C}$ for $10 \mathrm{~min}$. The samples were centrifuged for 5 minutes at $13,000 \mathrm{rpm}$ and $10 \mu \mathrm{L}$ of the supernatant was used for PCR. Firstly, the extracts were checked for DNA quality by standard amplification of the human B-globin, as described previously ${ }^{[21]}$. In B-globin positive samples, HPV detection was performed by the commercially available INNO-LiPA HPV detection/genotyping assay (SPF10-LiPA system version 2, Innogenetics, Belgium), based on the method outlined by Kleter et al. ${ }^{[22]}$. The PCR primers employed in this system amplify a 65-bp fragment of the L1 open reading frame of HPV genome. Each PCR experiment was performed with separate positive and several negative controls. By the current version of the INNOLiPA assay, 24 different HPV genotypes, of which 15 HR types (HPV-16, -18, -31, -33, -35, -39, -45, -51, $52,-53,-56,-58,-59,-66$ and-68) and 9 LR types (HPV-6, - 11, -40, -42, -43, -44, -54, -70 and -74) can be identified.

Statistical analysis. Analysis of associations between continuous and cathegorical variables was performed by means of Kendall's tau_b and Pearson chi-square tests. Alpha was assumed at a level of significance of 0.005 . Sensitivity, specificity and positive predictive values (PPV) were calculated for features found to be associated with the presence of HPV in univariate analysis and for the Mib1 index. The differences between HR-HPV and LR-HPV distribution in patients were assessed by the Fisher's exact test. All calculations were performed using the SPSS software package.

\section{RESULTS AND DISCUSSION}

The morphological features, Mib1 and HPV status of the 52 samples in study are shown in Table 1 . On the 
Am. J. Infect. Dis., 4 (3): 193-199, 2008

Table 1: Study cases by HPV status, histological feature and Mib-1 index

\begin{tabular}{|c|c|c|c|c|c|c|c|c|}
\hline HPV status & Koilocytosis & Binucleation & Acanthosis & Papillomatosis & Parakeratosis & Mitosis* & Distinc basal layer & Mib-1** \\
\hline Negative $(n=25)$ & 6 & 0 & 7 & 14 & 14 & 5 & 10 & 9 \\
\hline LR-HPV a $(n=9)$ & 6 & 1 & 1 & 4 & 2 & 2 & 1 & 1 \\
\hline HR-HPV b $(n=18)$ & 14 & 6 & 4 & 8 & 7 & 2 & 5 & 10 \\
\hline
\end{tabular}

$*$ = present in the 1/3 lower layer of the epithelium, ${ }^{*}=$ based on presence of at least two Mib1 positive cells in the upper two thirds of epithelium, $\mathrm{a}=$ Low-risk genotypes, $\mathrm{b}=$ High-risk genotypes

whole, koilocytosis was present in $26(50.0 \%)$ samples, binucleation in $7(13.5 \%)$, acanthosis in $12(23.0 \%)$, papillomatosis in $26(50.0 \%)$, parakeratosis in 23 $(44.2 \%)$, mitosis in the $1 / 3$ lower layer in $9(17.3 \%)$, distinct basal layer in $16(30.8 \%)$ and Mib1 expression in $20(38.5 \%)$ cases.

HPV was present in $27(52.0 \%)$ cases, as being 9 LR genotypes (HPV-6, -11 or-61) and 18 HR types, these latter present as either single (seven cases of HPV-16 or-18, two cases of each HPV-31, -33, -53 and -56 , one case of HPV-59) or mixed (one case of each HPV-11 and-33, HPV-6 and -53, HPV-39 and -53) HPV infections.

By univariate analysis, only koilocytosis and binucleation showed a significant association with HPV presence $(\mathrm{p}<0.001, \mathrm{R}=0.6)$. For any type HPV, koilocytosis had $74.0 \%$ sensitivity, $76.0 \%$ specificity and $77.0 \%$ PPV; for HR-HPV types, the corresponding values were $77.8,64.7$ and $53.8 \%$. Binucleation had very low sensitivity $(26.0 \%)$ for any type HPV, even though specificity and PPV were $100.0 \%$, for HR-HPV types, the corresponding values were 33.3, 97.0 and $85.7 \%$. The remaining morphologic features were not associated to either HPV or the two histological parameters (koilocytosis and binucleation) associated to HPV. The Mib1 index showed $40.7 \%$ sensitivity, $64.0 \%$, specificity and $55.0 \%$ PPV for any type HPV detection, for HR-HPV types, the corresponding values were $55.5,70.6$ and $50.0 \%$.

On the basis of HPV-DNA presence two groups were identifyed: HPV-DNA negative AL cases (25/52 cases, $48 \%)$ and HPV-DNA positive AL cases (27/52 cases. 52\%) When stratified by HPV results, koilocytosis and Mib-1 immunostaining, AL cases could be divided as follows:

HPV negative AL cases: Subgroup A: 11/25 cases, lacking of koilocytosis/multinucleation and of Mib-1 immunostaining. They could be considered normal and showed positivity for Mib1 immunostain only in a few nuclei of the parabasal layer (Fig. 1A).

Subgroup B: 7/25 cases, lacking of koilocytosis but showing mib-1 immunostain in the upper two thirds of the epithelium, indicating an increased proliferative activity. They were considered reactive/repair changes (Fig. 1B).

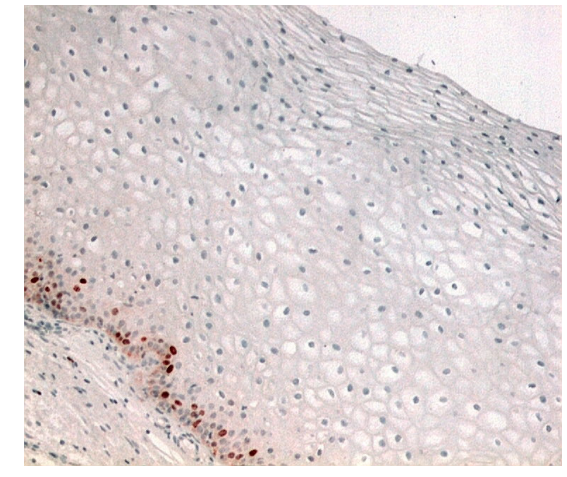

Fig. 1A: Normal epithelium: Absence of koilocytosis. Only parabasal nuclei were Mib1 positive

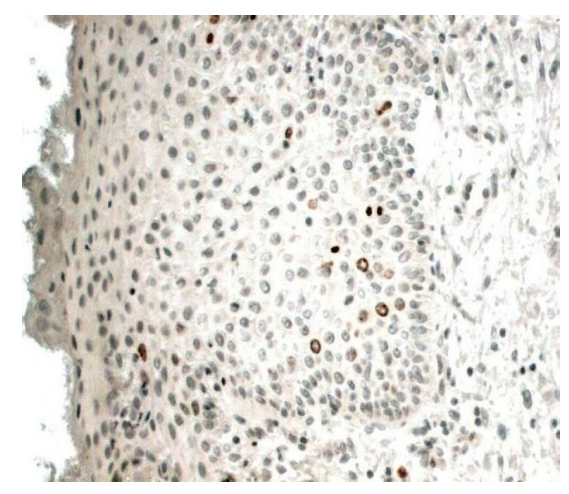

Fig. 1B: Metaplastic/reactive changes: Absence of koilocitosis and presence of Mib1 positive nuclei in intermediate layers

Subgroup C: $2 / 25$ cases, showing koilocytosis but lacking of multinucleation and of Mib1 positivity. They were considered mimics of koilocytosis(Fig. 1C).

subgroup D: 5/25 cases showing both koilocytosis and increased proliferative activity. They were considered doubtful cases, probably reactive/ repair change with mimics of koilocytosis or false negative of PCR -HPV detection.

HPV positive AL cases: Subgroup E: 7/27cases (3 with LR- HPV and 4 with HR- HPV), lacking of koilocytosis and of Mib1 positive immunostaining. They were considered latent, sub-clinical infection (Fig. 1D). 


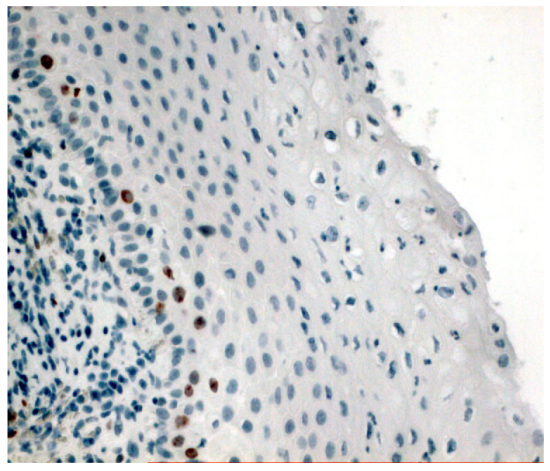

Fig. 1C: Mimics of koilocytosis. Koilocytosis was visible (as shown in the Ematoxylin-Eosin stained insert) but Mib1 positive cells were present only in the parabasal layers as in normal epithelium

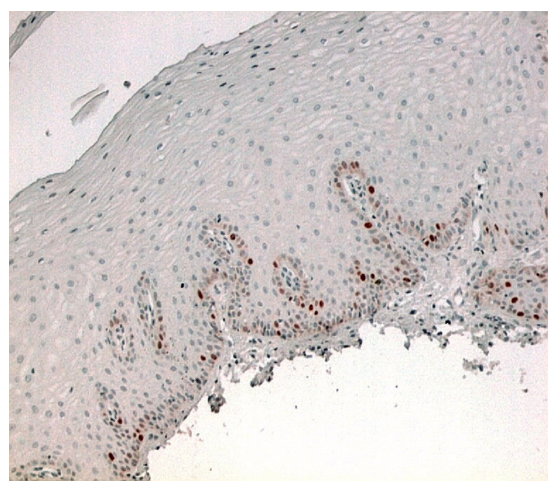

Fig. 1D: Latent HR-HPV infection (HPV 16) Absence of koilocytosis. Only parabasal nuclei were Mib1 positive, as in normal epithelium

Subgroup F: 9/27cases (5 of which with LR- HPV and 4 with HR- HPV) showing koilocytosis but lacking of Mib1 positivity. They were considered productive, cytopathic infection, without replicative activity (Fig. $1 \mathrm{E})$.

Subgroup G: $11 / 27$ cases (1 with LR-HPV types and 10 with HR-HPV types), showing both koilocytosis and proliferative activity. They were considered cases with active, proliferative infection (Fig. 1F).

No HPV positive cases showed increased proliferative activity without koilocytosis. The results are reported in Table 2.

Noteworthy, in the subgroup G only $1 / 11(9 \%)$ cases was LR-HPV whereas 10/11 (91\%) were HRHPV, with a statistically significative difference in the distribution of LR-HPV and HR-HPV types $(\mathrm{p}<0.005)$. On the contrary, there was no statistically significative

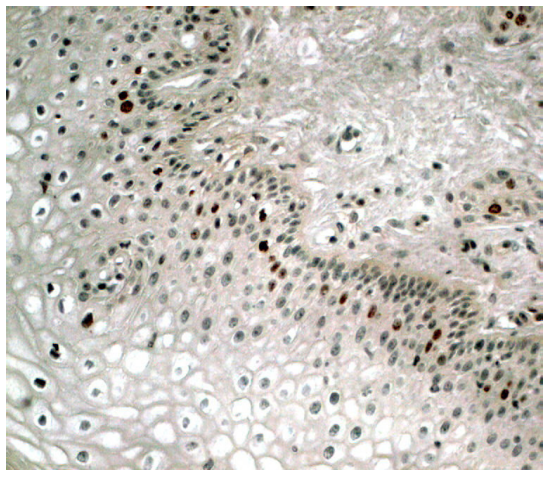

Fig. 1E: Cytopathic infection, without replicative activity (HPV 18). Koilocitosis was evident but Mib1 positive cells were present only in the parabasal layer, as in normal epithelium

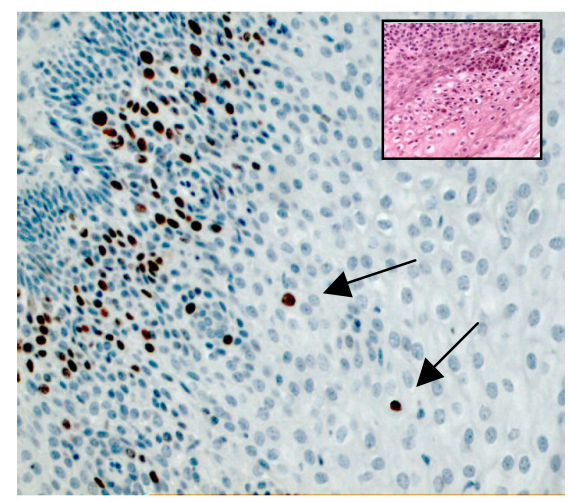

Fig. 1F: Proliferative infection (HPV16). Mild koylocitosis (shown in the Ematoxylin-Eosin stained insert) and pPresence of Mib1 positive nuclei in intermediate layers (arrows)

difference in the distribution of LR-HPV and HR-HPV types in the sub-groups $\mathrm{E}$ and $\mathrm{F}$, lacking of proliferative activity.

To improve the diagnosis and management of histological AL, it would be important to identify among them those lesions that are really HPVassociated and above all, those lesions harbouring HRHPV DNA. With this aim, the present analysis investigated the association between morphological features, proliferative activity assessed by Mib1 immunostaining and HPV status.

In cytological diagnosis, the introduction of a borderline category (atypical squamous cells of undetermined significance) of cervical lesions has been widely accepted ${ }^{[16]}$. In contrast, the introduction of a borderline category in histological diagnosis, as being either basal abnormalities of uncertain significance ${ }^{[8,11]}$ 
Am. J. Infect. Dis., 4 (3): 193-199, 2008

Table 2: AL subgroups in relation to HPV presence, koilocytosis and proliferative activity (based on Mib1 index)

\begin{tabular}{|c|c|c|c|}
\hline Total cases $=52$ & koilocytosis & Proliferative activity & Interpretation of sub-groups \\
\hline \multicolumn{4}{|l|}{ HPV Negative (25 cases) } \\
\hline 11 & - & - & A) normal \\
\hline 7 & - & + & B) reactive/repair changes \\
\hline 2 & + & - & C) Mimics of koilocytosis \\
\hline 5 & + & + & D) Doubtful cases $\wedge^{\wedge}$ \\
\hline \multicolumn{4}{|l|}{ HPV Positive ( 27 cases) * } \\
\hline 7 (3 LR-HPV) (4 HR-HPV) & - & - & E) Latent,sub-clinical infection \\
\hline 9 (5 LR-HPV) (4 HR-HPV) & + & - & F)Productive,cytopatic infection \\
\hline 11 (1 LR-HPV) (10 HR-HPV) & + & + & G) Infection with increased proliferative activity \\
\hline
\end{tabular}

or borderline condiloma ${ }^{[12,13]}$ has been strongly criticised, because of the increased inter-observer disagreement and low reproducibility in distinguishing $\mathrm{AL}$ from normal tissue and low dysplasia ${ }^{[7,23]}$. Also by using the Bethesda system, the pathologist's intra- and inter-observer agreement in the LSIL assessment remains low ${ }^{[2-9]}$. Additionally, cervical reactive/metaplastic conditions and LSIL can also be contemporary present, making the diagnosis also more difficult. Of note, a clear trend to over-diagnose normal, metaplastic or reactive cervical biopsies as HPV associated LSIL has been reported, with therapeutic, reproductive, sexual and social consequences ${ }^{[19,24]}$.

In this study no association was found in $\mathrm{AL}$ between HPV status and most of the morphological features investigated, namely, hyperkeratosis, parakeratosis, acanthosis, papillomatosis, mitosis in the lower third of epithelium, hyperplasia of parabasal layers, absence of a distinct basal cell layer. The lack of an association between the absence of a distinct basal cell layer and HPV status is in keeping with precedent reports, which found that a normal basal cell layer is usually maintained both in borderline condyloma and in CIN $1^{[12,13]}$.

As far as koilocytosis was concerned, it was found to be only a relatively sensitive and specific parameter (sensitivity $74 \%$ and specificity of $72 \%$ ) for HPV infection. Differently, binucleation was confirmed as a very specific parameter (100\% specificity), since it was absent in HPV negative cases; its sensitivity, however, was very low (26\% only). Our results are in line with those of Abadi et al. ${ }^{[8]}$, who showed that koilocytosis and binucleation, even when applied with stringent criteria, reached $70 \%$ sensitivity and $66 \%$ specificity for HPV related LSIL.

On the basis of the relatively poor sensitivity and specificity of morphological features, the Mib1 immunostain method was applied to AL to distinguish HPV related AL from metaplastic/ reactive changes. Previous studies on LSIL stated that the presence of Mib1 positive cells in the upper two thirds of epithelium was indicative of HPV presence ${ }^{[19,20]}$ and, analogously, Mib1 immunostain into the surface half of the epithelium was shown in condylomas but not in normal and reactive epithelium ${ }^{[25]}$. In this analysis, the Mib1 index was not proven to be either a sensitive or a specific method (sensitivity $40.7 \%$ and specificity $64.0 \%$ ). These results are in agreement with previously reported considerations of Al-Saleh et al. ${ }^{[26]}$, who found an overlap between Ki 67 positive cells in LSIL and in squamous metaplasia and thus concluded that Ki 67 can differentiate LSIL from HSIL, but not LSIL from squamous metaplasia in every case. Similarly, Pirog ${ }^{[19]}$ underlined that Mib1 testing has several limitations, because Mib1 positive cells may be present in the upper layers of epithelium in the immature squamous epithelium, squamous metaplasia, or in an area of cervical erosion (repair) not associated with HPV presence. Finally, the link between over-expression of Mib1 and both inflammation and reactive/atypical changes, has been recently reported ${ }^{[18]}$.

Even though Mib1 in this analysis was not shown as an independent, reliable marker of HPV infection, it still provided useful information and, when used toghether with koylocitosis, it allowed to stratify AL in sub-groups, among whom, the subgroup G could have a true clinical rilevance because it probably represents active infection with increased proliferative activity and shows HR-HPV DNA in 10/11 (91\%) of cases. On the contrary, the subgroups A, B, C, E and F, lacked of one or both the markers and they could represent clinically not relevant cases. In fact group A, B and C, resulted negative for HPV-DNA and were considered as normal (group A), reactive/repair changes (group B) and mimics of koylocitosis (group C). On the other side, group $\mathrm{E}$ and $\mathrm{F}$, despite they were HPV-DNA positive (that was LR-HPV DNA in about half of the cases), they didn't show any replicative activity. So they probably represent latent HPV infection, (group E), or productive cytopatic, non replicative infection (group F) in which, after a proliferating phase, cells carrying HPV genomes are being eliminated by the immune 
response. In all the above described cases, the clinical consequences of underdiagnosis would be mild. They would be similar to the large proportion of relatively harmless CIN lesions that do not progress (or even spontaneously regress), in which a cytological followup appears reasonable and for whom previous studies hypothesized to introduce a borderline category that entails follow up without treatment ${ }^{[6]}$.

The clinical meaning of the group D, that showed both the two markers but was negative for HPV-DNA, remains doubtful. It could represent reactive/ repair change with mimics of koilocytosis or false negative PCR -HPV detection cases.

In conclusion, AL appear as a very heterogeneous group of lesions, in which Mib1 assessment alone is not sensitive and specific enough to distinguish HPV negative from HPV positive lesions. Koilocytosis and multinucleation, although poorly sensitive and specific, remain the only features associated to HPV presence. Nevertheless, the Mib1 together with koylocitosis, could be useful to identify cases with HPV infection and encreased proliferative activity that are at higher risk of harbouring HR-HPV DNA (group G). With this approach it could be possible to increase the accuracy of histological diagnosis and to obtain a more correct management of the patients.

\section{REFERENCES}

1. Richart, R.M., 1973. Cervical intraepithelial neoplasia. Pathol Annu., 8: 301-328.

2. Ismail, S.M., A.B. Colcough and J.S. Dinner et al., 1990 Reporting cervical intra-epithelial neoplasia (CIN): intra- and interpathologist variation and factors associated with disagreement. Histopathology, 16: 371-376.

3. Geng, L., D.C. Connolly and C. Isacson, 1999. Atypical immature metaplasia (AIM) of the cervix: Is it related to high-grade squa intraepithelial lesion (HSIL)? Hum. Pathol., 30: 345-351.

4. Robertson, A.J. J.M. Anderson and J.S. Beck, 1989. Observer variation in histopathological reporting of cervical biopsy specimens. J. Clin. Pathol., 42: 231-238.

5. Stoler, M.H. and M. Schiffman, 2001. Atypical squamous cells of undetermined significance lowgrade squamous intraepithelial lesion triage study (ALTS) group. Interobserver reproducibility of cervical cytologic and histologic interpretations: Realistic estimates from the ASCUS-LSIL triage study. Jama, 285: 1500-1505.
6. Ismail, S.M., A.B. Colcough and J.S. Dinner, 1989. Observer variation in histopathological diagnosis grading of cervical intraepithelial neoplasia. $\mathrm{Br}$ Med. J., 298: 707-710.

7. Creagh, T., J.E. Bridger and E. Kupek et al., 1995. Pathologist variation in reporting cervical borderline epithelial abnormalities and cervical intraepithelial neoplasia. J. Clin. Pathol., 48: 59-60.

8. Abadi, M.A., G.Y.F. Ho and R.D. Burk et al., 1998. Stringent criteria for histological diagnosis of koilocytosis fail to eliminate overdiagnosis of human papillomavirus infection and cervical intraepithelial neoplasia grade 1. Hum. Pathol., 29: 54-59.

9. Grenko, R.T., C.S. Abendroth and E.E. Frauenhoffer et al., 2000. Variance in the interpretation of cervical biopsy specimens obtained for atypical squamous cells of undetermined significance. Am. J. Clin. Pathol., 114: 735-40.

10. The 2001. Bethesda System: terminology for reporting results of cervical cytology. JAMA. 2002 Apr 24;287(16):2114-9. Review

11. Anderson, M.C., C.L. Brown and C.H. Buckley et al., 1991 Current views on cervical intraepithelial neoplasia. J. Clin. Pathol., 44: 969-978.

12. Yao, S. Fu, 2002. Benign and Malignant Epithelial Lesions of the Uterine Cervix. In: Pathology of the Uterine Cervix, Vagina and Vulva. Philadelphia, USA: Saunders, pp: 269-470.

13. Rosai, J., 2004. Female reproductive system In: Rosai and Ackerman's surgical pathology. 2: 14831766.

14. Koss, L.G. and D.R. Durfee, 1956. Unusual patterns of squamous epithelium of the uterine cervix: Cytologic-pathologic study of koilocytotic atypia. Ann. N.Y. Acad. Sci., 61: 1235-61.

15. Meisels, A., C. Morin, M. Casas-Cordero, 1982. Human papilloma virus infection of the uterine cervix. Int. J. Gynecol. Pathol., 1: 75-94.

16. Nayar, R. and S.O. Tabbara, 2003. Atypical squamous cells: update on current concepts. Clin. Lab. Med., 23: 605-632.

17. Keating, J.T., A. Cviko and S. Riethdorf et al.. 2001. Ki-67, cyclin E and p16INK4a are complimentary surrogate biomarkers for human papilloma virus-related cervical neoplasia. Am. J. Surg. Pathol., 25: 884-891.

18. Agoff, S.N., P. Lin and J. Morihara et al., 2003. p16INK4a expression correlates with degree of cervical neoplasia: A comparison with Ki-67 expression and detection of high-risk HPV types. Mod. Pathol., 16: 665-673. 
19. Pirog, E.C., R.N. Baergen and R.A. Soslow, et al., 2002. Diagnostic accuracy of cervical low-grade squamous intraepithelial lesions is improved with MIB-1 immunostaining. Am. J. Surg. Pathol., 26: 70-75.

20. Pirog, E.C., Y.T. Chen and C. Isacson, 2000. MIB1 immunostaining is a beneficial adjunct test for accurate diagnosis of vulvar condyloma acuminatum. Am. J. Surg. Pathol., 24: 1393-1399.

21. Giovannelli, L., G. Campisi and A. Lama et al., 2002. Human papillomavirus DNA in oral mucosal lesions. J Infect Dis., 185: 833-836.

22. Kleter, B., L.J. van Doorn and J. ter Schegget et al., 1998 Novel short-fragment PCR assay for highly sensitive broad-spectrum detection of anogenital human papillomaviruses. Am. J. Pathol., 153: 1731-1739.

23. McCluggage W.G., H. Bharucha and L.M. Caughley et al., 1996 Interobserver variation in the reporting of cervical colposcopic biopsy specimens: Comparison of grading systems. J. Clin. Pathol., 49: 833-835.
24. Kruse, A.J., J.P.A. Baak and T. Helliesen et al., 2002. Evaluation of MIB-1 positive cell clusters as a diagnostic marker for cervical intraepithelial neoplasia. Am. J. Surg. Pathol., 26: 1501-1507.

25. Mittal, K. and J. Palazzo, 1998 Cervical condylomas show higher proliferation than do inflamed or metaplastic cervical squamous epithelium. Mod. Pathol., 11: 780-783.

26. Al-Saleh, W., P. Delvenne and R. Greimers et al., 1995. Assessment of Ki-67 antigen immunostaining in squamous intraepithelial lesions of the uterine cervix. Correlation with the histologic grade and human papillomavirus type. Anat. Pathol., 104: 154-160. 